\title{
IDENTIDAD Y ABUSO DE DROGAS EN ADOLESCENTES
}

\section{Identity and drug abuse in adolescents}

\author{
Miguel Angel Molla Madueño*
}

\begin{abstract}
Resumen
En el presente estudio se llevó a cabo una evaluación de la identidad de adolescentes, sobre la base de la teoría evolutiva de Erik Erikson. La muestra estaba formada por 68 hombres y mujeres adolescentes, 40 estudiantes en un Instituto de Secundaria y 28 en un programa terapéutico para adolescentes con problema de drogas, en Madrid, España. El objetivo del estudio fue comprender los factores que participan en la conformación de la identidad personal y su crisis. El estudio postula que el desarrollo de la identidad personal sería favorecido por la capacidad de aprender acerca de sí mismo y comprender su propio proceso de identidad.

Los resultados muestran que el desarrollo de la identidad personal se pone en riesgo por la presencia de los conflictos en la integración de nuevas experiencias emocionales. El total de la escala de la identidad, el aprendizaje y la laboriosidad, se ven afectados por un bajo logro de la perspectiva temporal y la falta de integración y asimilación de experiencias. La perspectiva temporal se consideró un elemento clave para la prevención del uso indebido de drogas entre los adolescentes en nuestra muestra. Se concluye que este es el factor que incide en forma más significativa a favor de la Identidad en ambos grupos, ya la perspectiva temporal conlleva la integración emocional-vital del sentido existencial o búsqueda de significado en la vida.
\end{abstract}

Palabras clave: identidad, consumo de drogas, adolescentes

\begin{abstract}
In the present study evaluation of the identity in adolescents was carried out, on the base of Erik Erikson's evolutionary theory. The sample was made by 68 male and female adolescents, 40 students from Secondary Institution and 28 from a therapeutic program for adolescents with drug problems, from Madrid, Spain. The objective of the study was to understand the factors participating in personal identity and his crisis. The study mentions that personal identity development will be benefited by the ability to learn about oneself and to understand the own identity process.

The results show that personal identity development jeopardizes by the presence of conflicts in the integration of new emotional experiences. The total of the identity, learning and laboriousness' scale is affected for low achievement of temporal perspective and the lack of integration as well as assimilation of experiences. The temporal perspective was considered as a key element for the prevention of the improper drug use among the adolescents in our sample. We can conclude that this factor which falls in a more significant aspect in favor of Identity in both groups and to temporal perspective, leads to the emotional-vital integration of existential meaning or the search for the meaning of life.
\end{abstract}

Key words: Identity, drug consumption, adolescents

* Psicólogo. Director del Centro de Logoterapia de Lima, Perú. migmolla@gmail.com 


\section{ANTECEDENTES y JUSTIFICACIÓN}

La presente investigación, se plantea en el campo de la problemática de la búsqueda de identidad, y está dirigida a caracterizar una población objetivo de adolescentes para identificar los factores predictores de una sana identidad y los problemas que los pueden hacer propensos al consumo de drogas. Para obtener su perfil, se busca conocer los procesos de formación de la identidad, por ser logros claves para el desarrollo humano. El estudio de la identidad con indicadores permitirá contar con una base de datos que facilite el éxito de programas de prevención y de tratamiento

\section{FUNDAMENTACIÓN TEÓRICA}

La identidad es un tema obligado para tratar la adolescencia, sus crisis y sus grandes posibilidades en el desarrollo humano, en lo intelectual, afectivo y en el desarrollo de valores y sociabilidad.

Es necesario encontrar la facilitación recíproca entre el desarrollo de la identidad individual y el desarrollo social y de una "inteligencia emocional" y social.

Los jóvenes de diversas sociedades atraviesan por dificultades cada vez mayores, debido a la presión de los problemas del complejo mundo moderno y de las a veces contradictorias exigencias del entorno social. En otros casos la frustración de sus expectativas los llevará a conductas negativas como expresión de su malestar que aumentaría su confusión de identidad.

Erik Erikson, $(1950,1968)$, ha desarrollado las más importantes aproximaciones al tema del desarrollo humano y la identidad del adolescente. El instrumento de evaluación o Test usado en esta investigación, ha sido creado sobre la base de esta teoría. Este Test se usa para aproximarnos a la problemática del adolescente, y evaluar su identidad.

La formación de la identidad sana, debemos tomar en cuenta todo el conjunto de procesos, tanto biológico como psicológico y social que crean una corriente única llamada fisiología del vivir. La Adolescencia llegará a su fin cuando un sólido sentimiento de identidad interior condicione una maduración más amplia y verdaderamente individual.

\section{PLANTEAMIENTO DEL ESTUDIO}

\section{Objetivos de la Investigación}

El objetivo de la presente investigación es la evaluación de identidad de un grupo de adolescentes pertenecientes a un programa de tratamiento por problema de drogas, comparándola con un grupo de adolescentes de una escuela secundaria, con la finalidad de obtener indicadores de la problemática y su prevención.

En este sentido, nuestro estudio busca conocer la conformación de la identidad personal y como ésta se vería favorecida por la capacidad de aprender sobre sí mismo. Esto es, entender la propia identidad, como sustrato de esta experiencia, logrando encausar la propia afectividad y desarrollando la perspectiva vital o perspectiva temporal, una inteligencia emocional sobre su propia existencia. Dificultarían este desarrollo de identidad personal, la presencia de conflictos mayores en la integración de nuevas experiencias emocionales.

En el presente estudio, aplicamos el Test de Identidad, elaborado sobre la base de la Teoría de Erik Erikson $(1950,1968)$. Este Test de Identidad fue construido por Molla, en base a esa teoría, analizando y elaborando indicadores en forma de preguntas, sobre las características esenciales que definen cada área del desarrollo hasta llegar a la quinta Etapa del desarrollo evolutivo propuesto por Erik Erikson, el de la identidad.

El Test de Identidad es un inventario con 110 reactivos que exploran los sentimientos conscientes que tienen los adolescentes sobre las características de su propia identidad. Aun cuando este proceso de identidad es en su mayor parte insconsciente, sus manifestaciones llegan a la conciencia del adolescente el cual tiene la capacidad de reconocerse a sí mismo y analizarse. Los factores que evalúa el Test son los siguientes:

- La Confianza Básica, confianza en sí mismo y en los demás; madurez, espontaneidad, flexibilidad y sentimiento de satisfacción.

- La Autonomía, sentido de organización de la vida personal como algo propio y de lo cual se es responsable; sentimiento de la propia adecuación. 
- La Iniciativa, expresión espontánea y segura de sí mismo; originalidad, capacidad de hacer decisiones, actividad y sociabilidad.

- La Laboriosidad, significa principalmente sentido de organización, y responsabilidad en el trabajo, decisión de resolver problemas, y de adaptarse socialmente.

- La Perspectiva Temporal, orientación en el tiempo y en el espacio personal-vital, asimilación de la experiencia con un aprovechamiento adecuado de los aspectos del intercambio interpersonal y de la sensibilidad (experiencia emocional).

- La Seguridad en Sí Mismo, sentimiento de la propia confiabilidad, sentido de la adecuación y organización de la vida personal.

- La Experimentación con el Rol, énfasis en la acción; el enfrentamiento de situaciones diversas; y ejercicio de anticipación de metas.

- El Aprendizaje, significa principalmente interés por el contacto con el medio ambiente y una estrategia del aprendizaje vital.

- Polarización Sexual: Adecuado grado de desarrollo del propio interés sexual.

- Liderazgo y Adhesión: Adecuada integración al grupo de "pares".

- El Compromiso Ideológico, orientación valorativa y participación en el ambiente.

Asimismo, se construyó un Cuestionario en el que se busca información sobre aquellos factores que pueden influir positivamente en la realización del logro de la identidad o que pueden afectar críticamente la Identidad, con preguntas sobre cuatro áreas:

1) Área Personal: Preguntas referidas a aspectos de sí mismo y de información general.

2) Área Familiar: Preguntas referidas a la familia, y situaciones de posibles conflictos ocurridos durante la infancia o la adolescencia.
3) Área Educacional: Preguntas referidas al colegio, profesores, compañeros de estudio.

4) Área Social: Preguntas referidas a la "Vida Social", identificación o adhesión social, religiosa, y política.

Finalmente se elaboró un Cuestionario sobre el Consumo de Sustancias psicoactivas, (Tabaco, Alcohol y Drogas y frecuencia del consumo).

\section{ESTUDIOS SOBRE LA VALIDEZ DE CONSTRUCCIÓN DEL TEST DE IDENTIDAD}

El Test de identidad fue aplicado en España a 294 adolescentes de tres diferentes Escuelas Secundarias de Madrid, como parte de una Tesis Doctoral, consistente en la "Elaboración y Validación de un Instrumento para evaluar la Identidad del adolescente", ${ }^{1}$ obteniéndose altos indicadores de validez y confiabilidad.

Encontramos en los resultados que, se comprueba igualmente la adecuada integración y agrupación de los ítems (consistencia interna), obteniéndose un coeficiente Alfa de Cronbach de .917 Asimismo, se encontraron correlaciones entre el Test de Identidad y las áreas en la muestra de estudiantes españoles, con nivel de significación alta.

1 La "Elaboración y Validación de un Instrumento para evaluar la Identidad del adolescente", está inscrita como proyecto de tesis doctoral, de Miguel Molla en el Departamento de Psicología Evolutiva y de la Educación de la Universidad de Complutense de Madrid. 
Tabla 1

Correlaciones entre el Test de Identidad y las áreas en la muestra de estudiantes españoles, N=294

\begin{tabular}{|c|c|c|c|c|c|c|}
\hline & & $\begin{array}{l}\text { Egoid } \\
\text { identidad }\end{array}$ & $\begin{array}{c}\text { Área } \\
\text { personal }\end{array}$ & $\begin{array}{c}\text { Área } \\
\text { familiar }\end{array}$ & $\begin{array}{c}\text { Área } \\
\text { educativa }\end{array}$ & $\begin{array}{c}\text { Área motivación } \\
\text { al aprendizaje }\end{array}$ \\
\hline \multirow[t]{2}{*}{$\begin{array}{l}\text { Valor de } \\
\text { identidad }\end{array}$} & $\begin{array}{l}\text { Correlación } \\
\text { de Pearson }\end{array}$ & 1 & $.581(* *)$ & $.447(* *)$ & $.345(* *)$ & $.519(* *)$ \\
\hline & $\begin{array}{l}\text { Sig. } \\
\text { (bilateral) }\end{array}$ & & .000 & .000 & .000 & .000 \\
\hline \multirow[t]{2}{*}{$\begin{array}{l}\text { Área } \\
\text { personal }\end{array}$} & $\begin{array}{l}\text { Correlación } \\
\text { de Pearson }\end{array}$ & $.581(* *)$ & 1 & $.446(* *)$ & $.415(* *)$ & $.554(* *)$ \\
\hline & $\begin{array}{l}\text { Sig. } \\
\text { (bilateral) }\end{array}$ & .000 & & .000 & .000 & .000 \\
\hline \multirow[t]{2}{*}{$\begin{array}{l}\text { Área } \\
\text { familiar }\end{array}$} & $\begin{array}{l}\text { Correlación } \\
\text { de Pearson }\end{array}$ & $.447(* *)$ & $.446(* *)$ & 1 & $.360(* *)$ & $.394(* *)$ \\
\hline & $\begin{array}{l}\text { Sig. } \\
\text { (bilateral) }\end{array}$ & .000 & .000 & & .000 & .000 \\
\hline \multirow[t]{2}{*}{$\begin{array}{l}\text { Área } \\
\text { educativa }\end{array}$} & $\begin{array}{l}\text { Correlación } \\
\text { de Pearson }\end{array}$ & $.345(* *)$ & $.415(* *)$ & $.360(* *)$ & 1 & $.441(* *)$ \\
\hline & $\begin{array}{l}\text { Sig. } \\
\text { (bilateral) }\end{array}$ & .000 & .000 & .000 & & .000 \\
\hline \multirow[t]{3}{*}{$\begin{array}{l}\text { Área motivación } \\
\text { al aprendizaje }{ }^{2}\end{array}$} & $\begin{array}{l}\text { Correlación } \\
\text { de Pearson }\end{array}$ & $.519(* *)$ & $.554(* *)$ & $.394(* *)$ & $.441(* *)$ & 1 \\
\hline & $\begin{array}{l}\text { Sig. } \\
\text { (bilateral) }\end{array}$ & .000 & .000 & .000 & .000 & \\
\hline & $\mathrm{N}$ & 294 & 294 & 294 & 294 & 294 \\
\hline
\end{tabular}

** La correlación es significativa al nivel 0,01 (bilateral).

Otros estudios se han conducido con adolescentes, jóvenes y adultos, en investigaciones y estudio de casos realizados en el Perú, en una experiencia clínica y profesional, desarrollada durante más de veinte años desde la elaboración del Test de Identidad en tesis de psicología en la Pontificia Universidad Católica del Perú.

Estos diversos estudios, confirman la alta confiabilidad y verifican la validez de contenido del
Test con respecto a adolescentes y jóvenes. El Test se creo originalmente con una muestra de adolescentes peruanos, y se ha verificado su validez de contenido, correlacionándolo con el Test de la Personalidad de Minnesota, M.M.P.I. y otros procedimientos de verificación de contenido. Asimismo, en las pruebas de diseño original, se encontró una adecuada confiabilidad, con un coeficiente .93

2 El área de motivación al aprendizaje, busca conocer la influencia de factores de motivación y compromiso con el estudio y estrategias de aprendizaje autónomo. 
Tabla 2

Correlaciones entre los Puntajes del Test de Identidad y las Escalas clínicas del Test Multifásico de la Personalidad de Minnesota, M.M.P.I. (Tesis original de Elaboración del Test de Identidad: N=61)

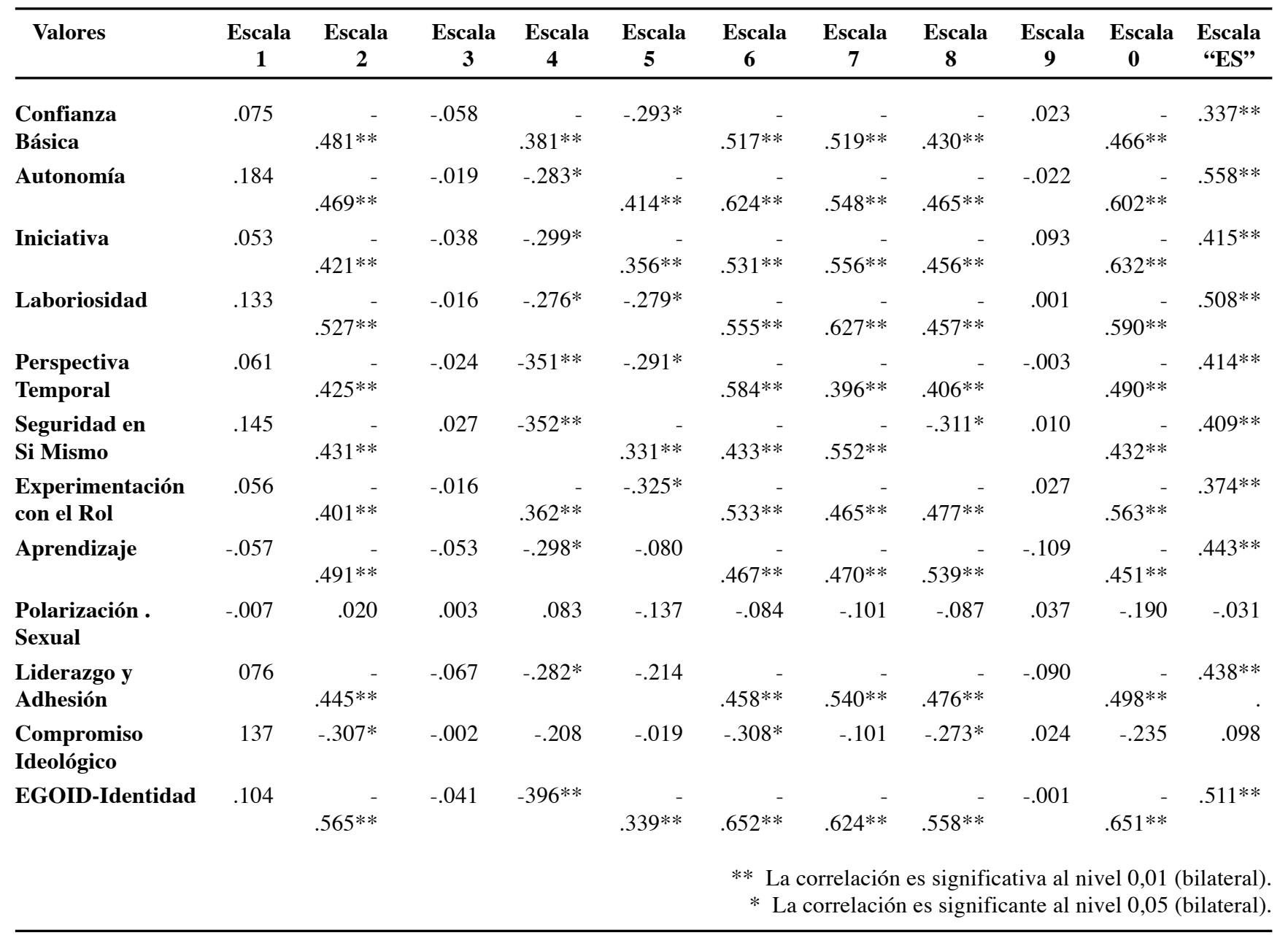

Leyenda.- Escalas clínicas del Inventario Multifásico de la Personalidad de Minnesota -M.M.P.I.

Escala 1-Hipocondría; Escala 2- Depresión; Escala 3-Histeria; Escala 4 -psicopatía; Escala 5-Masculino-Femenino; Escala 6 -Paranoia; Escala 7- Psicastenia (obsesivo-fóbicos); Escala 8- Esquizofrenia; Escala 9-Manía. La escala 0 de Introversión- Extroversión Social y la Escala en experimentación ES- Fortaleza Yoica.

El Test ha sido puesto a prueba aplicándose junto con una batería de otros test tanto de orientación vocacional como para fines de diagnóstico clínico en varios cientos de jóvenes. Siempre se han obtenido resultados coherentes con la validez de contenido, cruzando la información con los otros test.

En 1989, el Test fue sometido a un análisis minucioso, con una muestra de 354 jóvenes estudiantes de escuelas secundarias de Lima, en una investigación realizada con apoyo del Consejo Nacional de Ciencia y Tecnología del Perú, obteniéndose indicadores altos de confiabilidad, y mediante una Regresión Estadística, se obtiene un Coeficiente de determinación múltiple de .980 y Coeficiente de correlación múltiple de .9903 siendo la los valores de $\mathrm{p}=0.0000$ para todas las Escalas del Test.

En esa oportunidad se encontró asimismo, una alta correspondencia con las áreas personal, familiar, y una influencia de menor grado en las otras áreas educativa y social. 


\section{Gráfico 1 \\ Predictores de Identidad por áreas}
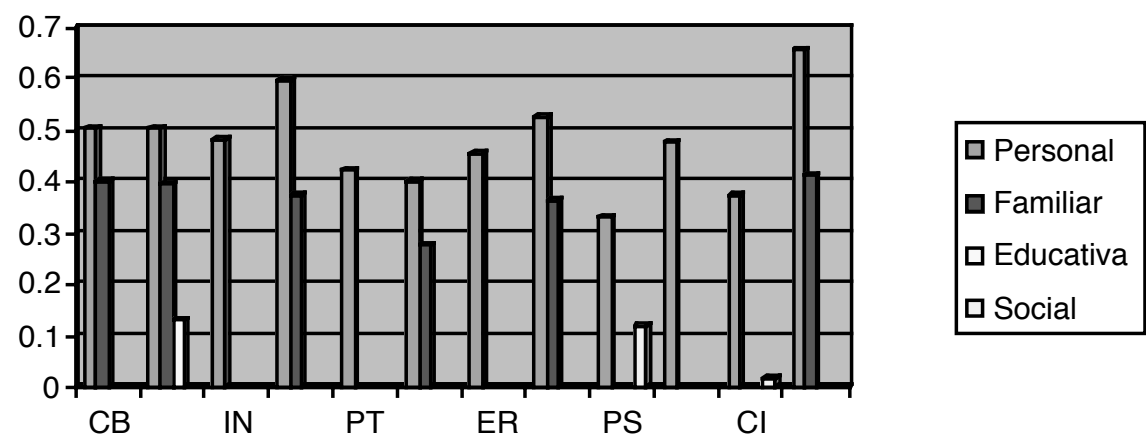

Asimismo, en otra nueva investigación en 1996, se revisó y adaptó el Test para aplicarlo a una muestra de mujeres con problema de drogas y crear un Perfil de las posibles usuarias de una Comunidad Terapéutica, a crearse en la ciudad de Lima. El estudio arrojó que el factor principal del deterioro de vida de las mujeres consumidoras era el trastorno de identidad disociativo. La muestra fue tomada de una población de mujeres adolescentes en régimen de tutela judicial y mujeres adultas en prisión, por lo cual, se tomó asimismo un grupo de comparación de mujeres supuestamente no consumidoras, siendo la muestra total de 93 mujeres, ver, Molla Miguel, y Raguz María: "Trastornos de identidad en mujeres con problema de drogas" $(\text { Lima })^{3}$

\section{METODOLOGÍA}

\section{Procedimientos}

Dado que nuestro estudio se interesa en los problemas de prevención al uso de drogas, tomamos una parte de la muestra en un programa de Comunidad Terapéutica para adolescentes con problema de drogas y poder así comparar las diversas problemáticas, desde su posible origen. La muestra de adolescentes es pequeña y no se pueden generalizar sus resultados, ya que se trata de un estudio exploratorio, cuyas conclusiones, en todo caso, solamente se van a circunscribir al grupo evaluado. Se toma otra parte de la muestra con estudiantes de Tercero de ESO, (secundaria), con fines de comparación, y por tratarse de un estudio que busca la prevención de problemas de identidad y de consumo en los adolescentes.

Muestra.- Está compuesta de la siguiente forma:

a) Adolescentes de un Programa de ayuda para superación de un problema de drogas: 28 adolescentes, (19 hombres y 9 mujeres).

b) Estudiantes de Institutos: 40 adolescentes, (21 hombres y 19 mujeres).

Los Adolescentes del Programa de ayuda pertenecen a una organización sin fines de lucro que brinda una gran variedad de programas de atención y apoyo a personas con problema de alcohol y drogas. Se ubica en la zona céntrica y cuenta con un amplio local que atiende al Programa de Menores en las tardes con actividades, grupos y terapia familiar.

Se escoge una muestra de adolescentes en Institutos por razón que la mayoría de los adolescentes realizan allí sus estudios escolares obligatorios, siendo además el Centro escogido uno ubicado en una zona de clase media y residencial de Madrid. No obstante ser un Centro Educativo normal y tener muy buenas

3 Investigación realizada y publicada con el auspicio Programa de las Naciones Unidas para la Fiscalización Internacional del Uso de Drogas, Lima 1997. 
condiciones de enseñanza, es lugar de concentración de diversas problemáticas, puestas en evidencia por los orientadores y profesores del mismo centro educativo, a la vez que es un recurso para los padres de familia ya que obtienen ayuda para sus hijos.

\section{Instrumentos}

Aparte del Test de Identidad, y del Cuestionario de las áreas Personal, Familiar, Educacional y Social, en el presente estudio, se diseñó y aplicó un Cuestionario sobre el Consumo de Sustancias psicoactivas, (Tabaco, Alcohol y Drogas y frecuencia del consumo). Las respuestas se clasifican de acuerdo a 3 grados de Consumo. Se computa un índice que va de 0 a 3,00 en el que se toma en cuenta la cantidad y frecuencia del uso y el tipo de estas substancias. Se valora como grado 1 un uso poco frecuente y poca cantidad o moderado; se valora como grado 2 un uso más frecuente y más fuera de control que el anterior. Se valora como grado 3, el abuso de sustancias como el que indica un cuadro de adicción de drogas. Se le da al tabaco un peso 1 , al alcohol un peso 2 y a drogas un peso 3. Siguiendo este criterio, la suma de estos índices da el factor «uso de sustancias psicoactivas» como un indicador del presente estudio.

\section{ANÁLISIS ESTADÍSTICO.}

El procesamiento y análisis estadístico de los datos e lleva a cabo con el paquete estadístico SPSS. Se obtienen las calificaciones individuales del test de identidad y se correlacionarán estos resultados con los datos sobre la situación en el área personal, familiar, educacional y social del Adolescente y su consumo de sustancias psicoactivas. Se comparan los resultados de los grupos de la muestra.

\section{Preguntas de Investigación}

Nos formulamos las siguientes preguntas de Investigación: ¿Cuáles son los Valores Psicométricos de los instrumentos del estudio? ¿Cuáles son las características sociodemográficas de los Adolescentes de la muestra? ¿Cuáles son las características de la Identidad? ¿Cuáles son las características de las Áreas Personal, Familiar, Educacional y Social? ¿Cuáles son las correlaciones entre la Identidad y las áreas? Los adolescentes del Programa de ayuda ¿en qué forma difieren de los adolescentes que estudian en Instituto, tanto en sus características de identidad como en las Áreas del estudio?

\section{RESULTADOS}

\section{Análisis Psicométrico de los Instrumentos de investigación}

Análisis Psicométrico del Test de Identidad: Se comprueba la validez de construcción de la prueba obteniéndose un coeficiente Alpha de confiabilidad del Test de Identidad, que es de .849 Igualmente se comprueba la adecuada integración y agrupación de los ítems (consistencia interna), obteniéndose los indicadores siguientes: $\mathrm{p}=0.0000$ para todas las Escalas, siendo las correlaciones entre las escalas del test adecuadamente altas. Aunque la muestra es relativamente pequeña el Test ha sido validado en otras investigaciones. Asimismo ha sido aplicado a una muestra mayor de estudiantes de escuelas secundarias de España. Estos resultados apoyan la validez del instrumento y su confiabilidad.

Análisis Psicométrico del Cuestionario de Áreas Personal, Familiar, Educacional y Social. El análisis revela una adecuada agrupación de los ítems y consistencia interna. Igualmente, las correlaciones entre los ítems son adecuadamente altas. El coeficiente de confiabilidad del Cuestionario es Alpha $=.6751$

\section{Análisis Psicométrico del Cuestionario de uso de sustancias psicoactivas}

El coeficiente de confiabilidad del Cuestionario es Alpha $=.8071$

\section{Características sociodemográficas y de perfil de vida de la muestra}

La mayoría de los adolescentes tienen entre 13 y 15 años, y toda la muestra del Instituto son estudiantes que cursan el $3^{\circ}$ de ESO. En cuanto al los adolescentes del Programa, sus edades van de 15 a 23 años, siendo su nivel educativo variado y va de $3^{\circ} \mathrm{ESO}$, hasta la Universidad, (en su mayoría no están actualmente estudiando). 


\section{Características de Identidad de los Adolescentes} de la muestra.

En la muestra, la edad, el género y grado de instrucción, no establecen diferencias importantes respecto al logro de la identidad. No obstante, se observa una correlación baja pero positiva de la edad, y el grado de instrucción con la identidad en estos jóvenes, que es significante al nivel 0.05 (bilateral).

\section{Análisis del perfil de Identidad de la muestra}

En el Test de Identidad, los adolescentes del Instituto presentan menores puntajes con respecto a los que obtienen los del Programa. Esto se explicaría en parte a que éstos están en un programa muy intensivo de trabajo sobre su personalidad (en el programa de terapia), y a que tienen una mayor media de edad.

Tabla 3

Puntajes en el Test de Identidad en Ambos Grupos

Estadísticos de grupo $\mathrm{N}=40$ instituto, $\mathrm{N}=28$ Programa

\begin{tabular}{llccc}
\hline & Centro & Media & $\begin{array}{c}\text { Desviación } \\
\text { típica. }\end{array}$ & $\begin{array}{c}\text { Error típico. } \\
\text { de la media }\end{array}$ \\
\hline Confianza básica & Instituto & 6.58 & 1.500 & .237 \\
Autonomía & Programa & 7.46 & 1.732 & .327 \\
Iniciativa & Instituto & 5.93 & 1.457 & .230 \\
Laboriosidad & Programa & 5.64 & 2.281 & .431 \\
Perspectiva Temporal & Instituto & 6.08 & 1.655 & .262 \\
& Programa & 6.71 & 1.675 & .316 \\
Seguridad en si mismo & Instituto & 6.93 & 1.966 & .311 \\
& Programa & 7.21 & 2.200 & .416 \\
Experimentación con el rol & Instituto & 6.00 & 2.309 & .365 \\
& Programa & 6.75 & 2.012 & .380 \\
Aprendizaje & Instituto & 6.08 & 1.789 & .283 \\
Polarización sexual & Programa & 6.86 & 1.737 & .328 \\
& Instituto & 6.05 & 1.449 & .229 \\
Liderazgo y adhesión & Programa & 6.79 & 2.114 & .400 \\
& Instituto & 6.93 & 2.117 & .335 \\
Compromiso ideológico & Programa & 7.54 & 2.333 & .441 \\
& Instituto & 7.23 & 1.776 & .281 \\
EGOID- identidad & Programa & 7.18 & 1.701 & .321 \\
& Instituto & 6.68 & 1.509 & .239 \\
& Programa & 7.36 & 1.367 & .258 \\
\hline & Instituto & 5.93 & 1.542 & .244 \\
& Programa & 6.79 & 2.061 & .390 \\
& Instituto & 6.3908 & 1.03519 & .16368 \\
& Programa & 6.9607 & 1.29780 & .24526 \\
\hline
\end{tabular}




\section{Gráfico 2:}

\section{Puntajes en el Test de Identidad en Ambos Grupos}

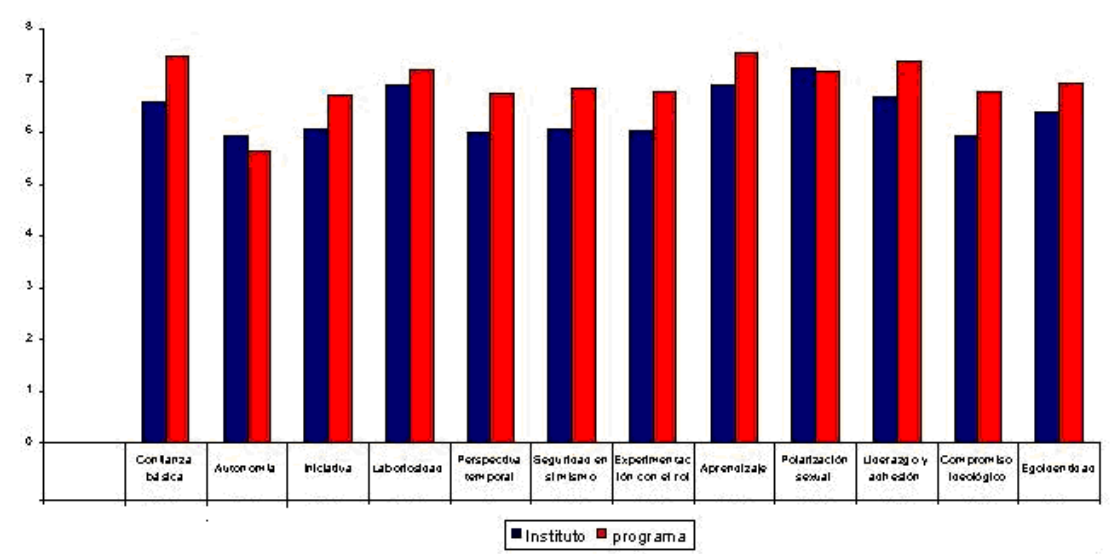

Tabla 4

Diferencias significativas entre los puntajes de Identidad del Grupo 1 Instituto, y del Grupo 2, Programa

\begin{tabular}{lrrc}
\hline Variable & F & Sig. & $\begin{array}{c}\text { Existen Diferencias } \\
\text { Significativas }\end{array}$ \\
\hline Confianza básica & 1.052 & .309 & \\
Autonomía & 7.374 & .008 & Grupo 1-2 \\
Iniciativa & .002 & .969 & \\
Laboriosidad & .718 & .400 & \\
Perspectiva temporal & .074 & .787 & Grupo 2-1 \\
Seguridad en sí mismo & .163 & .688 & \\
Experimentación con el rol & 6.980 & .010 & \\
Aprendizaje & .020 & .888 & \\
Polarización sexual & .004 & .949 & \\
Liderazgo y adhesión & .165 & .686 & \\
Compromiso ideológico & 2.128 & .149 & \\
\hline
\end{tabular}

Nota: Los valores de p igual o menor que 0.05 indican diferencias significativas.

Prosiguiendo nuestro análisis, encontramos que, respecto de la identidad de los adolescentes del programa y del Instituto secundario, no existen diferencias significativas entre estos puntajes, salvo en lo que respecta a la Autonomía (con una diferencia favorable a la muestra de adolescentes del Instituto) y en lo que respecta a la Experimentación con el Rol, (con una diferencia favorable a la muestra de adolescentes del Programa).
En cuanto a la primera diferencia significativa, se explica ya que los adolescentes del Instituto están en plena libertad y los del programa no, ya que tienen severas restricciones para salidas, uso del dinero y decisiones personales, todo debe ser consultado y aprobado por el programa antes de realizarse.

En cuanto a la segunda diferencia significativa, hay dos aspectos que lo explicarían, uno es la 
experiencia durante el consumo, la historia en la vida de los adolescentes del programa. Tienen muchas experiencias durante el consumo, han experimentado mucho más que los otros adolescentes, incluso en forma negativa. Y en la presente etapa, realizan múltiples actividades formativas.
Análisis del perfil respecto a las áreas personal, familiar, educativa y social.

Observamos mejores puntajes en todas las áreas a favor de la muestra de adolescentes del Instituto. No obstante, se verifica que no existen Diferencias significativas entre las medias de los puntajes de los grupos.

Tabla 5

Puntajes promedio en las Areas Personal, familiar, Educativa y Social Estadísticos de los grupos $\mathrm{N}=40$ instituto, $\mathrm{N}=28$ Programa

\begin{tabular}{lllcc}
\hline & Centro & Media & Desviación típica & Error típico de la media \\
\hline Personal & Instituto & 3.8658 & .52424 & .08289 \\
& Programa & 3.5811 & .54617 & .10322 \\
Familiar & Instituto & 3.9645 & .79413 & .12556 \\
& Programa & 3.4271 & .84337 & .15938 \\
Educacional & Instituto & 3.5223 & .63177 & .09989 \\
& Programa & 3.1321 & .79441 & .15013 \\
Social & Instituto & 3.0008 & .65419 & .10344 \\
& Programa & 2.7682 & .59780 & .11297 \\
\hline
\end{tabular}

\section{Gráfico 3:}

Puntajes promedio en las Áreas

Personal, familiar, Educativa y Social de ambos Grupos

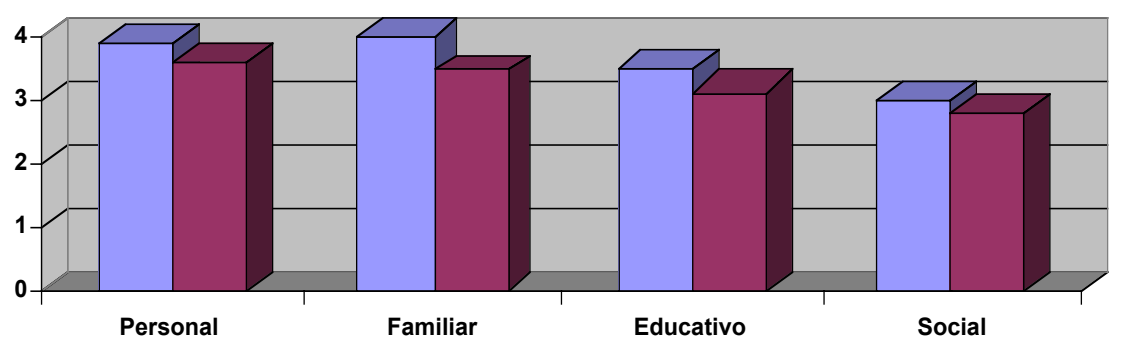


Asimismo, observamos que No existen diferencias significativas los grupos del Instituto y del Programa, en las áreas Personal, Familiar, Educativa y Social.

\section{Uso de sustancias psicoactivas}

Comparamos el Uso de Alcohol, Tabaco y Drogas en ambos grupos. En el Instituto la media del consumo es de ,48 mientras que en el Programa es de 2,34. Al cumplir los 15 años, en ambos grupos, la mayoría en la muestra total, ha hecho uso de algún tipo de droga que en promedio da un índice de .5200; (Hemos evaluado en los adolescentes del Programa de rehabilitación, no su uso actual sino el que hicieron antes, de estas sustancias, ya que actualmente están en abstinencia total).

Tabla 6

Promedios de uso de sustancias psicoactivas por nivel de edad

\begin{tabular}{cccc}
\hline Edad Años & Media & Desv. TPI. & Casos \\
\hline $\mathbf{1 3}$ & .0500 & .0707 & 2 \\
$\mathbf{1 4}$ & .5667 & .6364 & 9 \\
$\mathbf{1 5}$ & .5200 & .5239 & 24 \\
$\mathbf{1 6}$ & .7333 & .5888 & 6 \\
$\mathbf{1 7}$ & 1.5750 & 1.0145 & 4 \\
$\mathbf{1 8}$ & 2.3000 & .0000 & 2 \\
$\mathbf{1 9}$ & 2.5286 & .4348 & 7 \\
$\mathbf{2 0}$ & 2.3250 & .4349 & 4 \\
$\mathbf{2 1}$ & 2.6250 & .2872 & 4 \\
$\mathbf{2 2}$ & 2.4600 & .5941 & 5 \\
$\mathbf{2 3}$ & 2.3000 & & 1 \\
& & & Total $=68$ \\
\hline
\end{tabular}

Tabla 7

Valores de uso de sustancias piscoactivas en el Instituto y el Programa

\begin{tabular}{llcccc}
\hline Centro & N & Media & Mínimo & Máximo & Suma \\
\hline Instituto & 40 & .4870 & .00 & 1.90 & 19.48 \\
Programa CT & 28 & 2.3429 & 1.30 & 3.00 & 65.60 \\
\hline
\end{tabular}

El Uso de sustancias psicoativas tiene una alta correlación significativa, con estar en el Programa, edad y nivel de estudios 


\section{Análisis de indicadores sobre la Perspectiva Temporal.}

Los resultados de las correlaciones de la Perspectiva Temporal con las demás variables revelan la importancia de este factor para la identidad de los adolescentes evaluados.

Una débil Perspectiva Temporal denota estar afectados por la falta de integración vital-temporal o la "confusión del tiempo". Asimismo esto tiene relación con la desconfianza y los problemas de identidad. Si este factor no está bien logrado, pueden también alterarse otros mecanismos emocionales, con dificultad en asimilar nuevas experiencias que tienen los adolescentes. La "confusión del tiempo" afecta el mecanismo de aprendizaje e integración de nuevas experiencias emocionales.

Mediante una análisis de Regresión múltiple de la perspectiva temporal, con todas las otras variables de la identidad y las 4 áreas, como Variables predictoras: (Constante), encontramos un $\mathrm{R}$ cuadrado corregida de r2=4.95 y $\mathrm{F}=5.690$ y significatividad de .000

Tabla 8

Análisis de Correlaciones de la Perspectiva Temporal con los factores de la Identidad y las áreas personal, familiar, educativa y social, en la muestra total $(\mathrm{N}=68)$.

\begin{tabular}{lcc}
\hline Variable & Correlación & Sig. (bilateral) \\
\hline Confianza básica & $436^{* *}$ & .000 \\
Autonomía & $.307 *$ & .011 \\
Iniciativa & $374 * *$ & .002 \\
Laboriosidad & $.546^{* *}$ & .000 \\
Seguridad en si mismo & $.424 * *$ & .000 \\
Experimentación con el rol & $.409 * *$ & .001 \\
Aprendizaje & $.661 * *$ & .000 \\
Polarización sexual & .057 & .643 \\
Liderazgo y adhesión & $.265 *$ & .029 \\
Compromiso ideológico & .195 & .112 \\
Área personal & $.336^{* *}$ & .005 \\
Área familiar & $.263 *$ & .030 \\
Área Educativa & .010 & .938 \\
Área Social & $.258^{*}$ & .033 \\
\hline
\end{tabular}

Por lo anterior, La Perspectiva Temporal puede ser considerada un indicador para la prevención y para la orientación de los adolescentes que están estudiando en las escuelas. En los adolescentes de la muestra del Instituto, la Perspectiva Temporal tiene una correlación de -.476 respecto de problemas de abuso de drogas. A su vez, el problema del consumo de drogas se correlaciona negativamente con la identidad, afectaría la identidad en este grupo. Los adolescentes del Programa, ya han reforzado su identidad y su consumo no es actual, por ello no se puede notar el efecto negativo como podría suceder al inicio del consumo, o al aumentar este consumo de estar presente en adolescentes del Instituto. 


\section{Tabla 9}

Correlaciones de Pearson entre la perspectiva Temporal,

la identidad y el uso de sustancias psicoactivas, en el Instituto y en el Programa

\begin{tabular}{|c|c|c|c|c|c|}
\hline Centro & & & $\begin{array}{l}\text { Perspectiva } \\
\text { temporal }\end{array}$ & $\begin{array}{l}\text { Uso de sustancias } \\
\text { psicoactivas }\end{array}$ & $\begin{array}{l}\text { Identidad } \\
\text { (Egoid) }\end{array}$ \\
\hline \multirow[t]{7}{*}{ Instituto } & \multirow[t]{2}{*}{$\begin{array}{l}\text { Perspectiva } \\
\text { temporal }\end{array}$} & $\begin{array}{l}\text { Correlación de } \\
\text { Pearson }\end{array}$ & 1 & $-.476(* *)$ & $.710(* *)$ \\
\hline & & Sig. (bilateral) & & .002 & .000 \\
\hline & \multirow[t]{2}{*}{$\begin{array}{l}\text { Uso de sustancias } \\
\text { psicoactivas }\end{array}$} & $\begin{array}{l}\text { Correlación } \\
\text { de Pearson }\end{array}$ & $-.476(* *)$ & 1 & $-.316(*)$ \\
\hline & & Sig. (bilateral) & .002 & & .047 \\
\hline & \multirow[t]{3}{*}{$\begin{array}{l}\text { Identidad } \\
\text { (Egoid) }\end{array}$} & $\begin{array}{l}\text { Correlación } \\
\text { de Pearson }\end{array}$ & $.710(* *)$ & $-.316\left(^{*}\right)$ & 1 \\
\hline & & Sig. (bilateral) & .000 & .047 & \\
\hline & & $\mathbf{N}$ & 40 & 40 & 40 \\
\hline \multirow[t]{7}{*}{ Programa } & \multirow[t]{2}{*}{$\begin{array}{l}\text { Perspectiva } \\
\text { temporal }\end{array}$} & $\begin{array}{l}\text { Correlación } \\
\text { de Pearson }\end{array}$ & 1 & .112 & $.703(* *)$ \\
\hline & & Sig. (bilateral) & & .572 & .000 \\
\hline & \multirow[t]{2}{*}{$\begin{array}{l}\text { Uso de sustancias } \\
\text { psicoactivas }\end{array}$} & $\begin{array}{l}\text { Correlación } \\
\text { de Pearson }\end{array}$ & .112 & 1 & .032 \\
\hline & & Sig. (bilateral) & .572 & & .872 \\
\hline & \multirow[t]{3}{*}{$\begin{array}{l}\text { Identidad } \\
\text { (Egoid) }\end{array}$} & $\begin{array}{l}\text { Correlación } \\
\text { de Pearson }\end{array}$ & $.703(* *)$ & .032 & 1 \\
\hline & & Sig. (bilateral) & .000 & .872 & \\
\hline & & $\mathbf{N}$ & 28 & 28 & 28 \\
\hline
\end{tabular}

** La correlación es significativa al nivel 0,01 (bilateral).

* La correlación es significante al nivel 0,05 (bilateral) 
Tabla 10

Correlaciones del uso de sustancias psicoativas con algunas escalas de la Identidad en el Instituto, N=40

\begin{tabular}{|c|c|c|c|c|c|c|c|c|}
\hline & $\begin{array}{c}\text { Perspectiva } \\
\text { temporal }\end{array}$ & $\begin{array}{l}\text { Confianza } \\
\text { básica }\end{array}$ & Autonomia & Laboriosidad & Aprendizaje & $\begin{array}{l}\text { Exp. con } \\
\text { el rol }\end{array}$ & EGOID & $\begin{array}{c}\text { Uso de } \\
\text { sustancias } \\
\text { psico-activas }\end{array}$ \\
\hline $\begin{array}{l}\text { Perspectiva } \\
\text { temporal }\end{array}$ & 1 & $.474(* *)$ & .221 & $.576(* *)$ & $.677(* *)$ & $.452(* *)$ & $.710(* *)$ & $-.476(* *)$ \\
\hline Sig. (bilateral) & & .002 & .171 & .000 & .000 & .003 & .000 & .002 \\
\hline $\mathbf{N}$ & & 40 & 40 & 40 & 40 & 40 & 40 & 40 \\
\hline $\begin{array}{l}\text { Confianza } \\
\text { básica }\end{array}$ & $.474(* *)$ & 1 & .255 & $.458(* *)$ & $.482(* *)$ & $.435(* *)$ & $.645(* *)$ & -.243 \\
\hline Sig. (bilateral) & .002 & & .112 & .003 & .002 & .005 & .000 & .130 \\
\hline $\mathbf{N}$ & 40 & 40 & 40 & 40 & 40 & 40 & 40 & 40 \\
\hline Autonomia & .221 & .255 & 1 & .195 & .231 & -.010 & $.408(* *)$ & .071 \\
\hline Sig. (bilateral) & .171 & .112 & & .228 & .152 & .950 & .009 & .664 \\
\hline $\mathbf{N}$ & 40 & 40 & 40 & 40 & 40 & 40 & 40 & 40 \\
\hline Laboriosidad & $.576(* *)$ & $.458(* *)$ & .195 & 1 & $.615(* *)$ & .235 & $.715(* *)$ & -.191 \\
\hline Sig. (bilateral) & .000 & .003 & .228 & & .000 & .144 & .000 & .238 \\
\hline $\mathbf{N}$ & 40 & 40 & 40 & 40 & 40 & 40 & 40 & 40 \\
\hline Aprendizaje & $.677(* *)$ & $.482(* *)$ & .231 & $.615(* *)$ & 1 & $.428(* *)$ & $.855(* *)$ & $-.372(*)$ \\
\hline Sig. (bilateral) & .000 & .002 & .152 & .000 & & .006 & .000 & .018 \\
\hline $\mathbf{N}$ & 40 & 40 & 40 & 40 & 40 & 40 & 40 & 40 \\
\hline $\begin{array}{l}\text { Experimentacion } \\
\text { con el rol }\end{array}$ & $.452(* *)$ & $.435(* *)$ & -.010 & .235 & $.428(* *)$ & 1 & $.530(* *)$ & -.122 \\
\hline Sig. (bilateral) & .003 & .005 & .950 & .144 & .006 & & .000 & .452 \\
\hline $\mathbf{N}$ & 40 & 40 & 40 & 40 & 40 & 40 & 40 & 40 \\
\hline $\begin{array}{l}\text { Valor de identidad } \\
\text { del sujeto }\end{array}$ & $.710(* *)$ & $.645(* *)$ & $.408(* *)$ & $.715(* *)$ & $.855(* *)$ & $.530(* *)$ & $1.316(*)$ & \\
\hline Sig. (bilateral) & .000 & .000 & .009 & .000 & .000 & .000 & & .047 \\
\hline $\mathbf{N}$ & 40 & 40 & 40 & 40 & 40 & 40 & 40 & 40 \\
\hline $\begin{array}{l}\text { Uso de sustancias } \\
\text { psicoactivas }\end{array}$ & $-.476(* *)$ & -.243 & .071 & -.191 & $-.372(*)$ & -.122 & $-.316(*)$ & 1 \\
\hline Sig. (bilateral) & .002 & .130 & .664 & .238 & .018 & .452 & .047 & \\
\hline $\mathrm{N}$ & 40 & 40 & 40 & 40 & 40 & 40 & 40 & 40 \\
\hline
\end{tabular}

** La correlación es significativa al nivel 0,01 (bilateral).

* La correlación es significante al nivel 0,05 (bilateral). 
Tabla 11

Correlaciones del uso que se hizo en el pasado, de sustancias psicoativas, con algunas escalas de la Identidad en el Programa

\begin{tabular}{|c|c|c|c|c|c|c|c|c|}
\hline & $\begin{array}{l}\text { Perspectiva } \\
\text { temporal }\end{array}$ & $\begin{array}{l}\text { Confianza } \\
\text { básica }\end{array}$ & Autonomía & Laboriosidad & Aprendizaje & $\begin{array}{l}\text { Exp. con } \\
\text { el rol }\end{array}$ & EGOID & $\begin{array}{c}\text { Uso de } \\
\text { sustancias } \\
\text { psico-activas }\end{array}$ \\
\hline $\begin{array}{l}\text { Perspectiva } \\
\text { temporal }\end{array}$ & 1 & .332 & $.464(*)$ & $.506(* *)$ & $.629(* *)$ & .344 & $.703(* *)$ & .112 \\
\hline Sig. (bilateral) & & .084 & .013 & .006 & .000 & .073 & .000 & .572 \\
\hline $\mathrm{N}$ & 28 & 28 & 28 & 28 & 28 & 28 & 28 & 28 \\
\hline $\begin{array}{l}\text { Confianza } \\
\text { básica }\end{array}$ & .332 & 1 & $.419(*)$ & $.605(* *)$ & $.395(*)$ & $.382(*)$ & $.655(* *)$ & .261 \\
\hline Sig. (bilateral) & .084 & & .027 & .001 & .038 & .045 & .000 & .180 \\
\hline $\mathrm{N}$ & 28 & 28 & 28 & 28 & 28 & 28 & 28 & 28 \\
\hline Autonomía & $.464(*)$ & $.419(*)$ & 1 & $.584(* *)$ & .351 & $.421(*)$ & $.749(* *)$ & -.045 \\
\hline Sig. (bilateral) & .013 & .027 & & .001 & .067 & .026 & .000 & .819 \\
\hline $\mathrm{N}$ & 28 & 28 & 28 & 28 & 28 & 28 & 28 & 28 \\
\hline Laboriosidad & $.506(* *)$ & $.605(* *)$ & $.584(* *)$ & 1 & $.698(* *)$ & $.392(*)$ & $.785(* *)$ & .256 \\
\hline Sig. (bilateral) & .006 & .001 & .001 & & .000 & .039 & .000 & .188 \\
\hline $\mathrm{N}$ & 28 & 28 & 28 & 28 & 28 & 28 & 28 & 28 \\
\hline Aprendizaje & $.629(* *)$ & $.395(*)$ & .351 & $.698(* *)$ & 1 & $.490(* *)$ & $.732(* *)$ & .155 \\
\hline Sig. (bilateral) & .000 & .038 & .067 & .000 & & .008 & .000 & .431 \\
\hline $\mathrm{N}$ & 28 & 28 & 28 & 28 & 28 & 28 & 28 & 28 \\
\hline $\begin{array}{l}\text { Experimentacion } \\
\text { con el rol }\end{array}$ & .344 & $.382(*)$ & $.421(*)$ & $.392(*)$ & $.490(* *)$ & 1 & $.619(* *)$ & .115 \\
\hline Sig. (bilateral) & .073 & .045 & .026 & .039 & .008 & & .000 & .561 \\
\hline $\mathrm{N}$ & 28 & 28 & 28 & 28 & 28 & 28 & 28 & 28 \\
\hline $\begin{array}{l}\text { Valor de identidad } \\
\text { del sujeto }\end{array}$ & $.703(* *)$ & $.655(* *)$ & $.749(* *)$ & $.785(* *)$ & $.732(* *)$ & $.619(* *)$ & 1 & .032 \\
\hline Sig. (bilateral) & .000 & .000 & .000 & .000 & .000 & .000 & & .872 \\
\hline $\mathrm{N}$ & 28 & 28 & 28 & 28 & 28 & 28 & 28 & 28 \\
\hline $\begin{array}{l}\text { Uso de sustancias } \\
\text { psicoactivas }\end{array}$ & .112 & .261 & -.045 & .256 & .155 & .115 & .032 & 1 \\
\hline Sig. (bilateral) & .572 & .180 & .819 & .188 & .431 & .561 & .872 & \\
\hline $\mathrm{N}$ & 28 & 28 & 28 & 28 & 28 & 28 & 28 & 28 \\
\hline
\end{tabular}

** La correlación es significativa al nivel 0,01 (bilateral).

* La correlacón es significante al nivel 0,05 (bilateral).

En los adolescentes del Instituto, el uso de sustancias se relaciona en forma negativa con la identidad. A mayor uso de sustancias, menor identidad. La correlación de -.316 es significativa al nivel del 0,05 . En los adolescentes del programa, por estar en terapia, el uso de sustancias no es actual y por ello no representa amenaza para la identidad, es decir, tiene una correlación muy baja con identidad. Respecto a la Perspectiva Temporal, está asociada en forma alta y significativa con la identidad y tiene una fuerte asociación con el desarrollo de sus diversos componentes, implicaría un buen ajuste afectivo y emocional y la integración de lo cognitivo-racional, para el aprendizaje vital y la identidad. 


\section{CONCLUSIONES}

1. Se ha podido confirmar la validez y confiabilidad de los instrumentos aplicados, instrumentos con los que es posible obtener indicadores de la identidad, para conocer problemáticas en forma clara y precisa.

2. Los resultados presentes solo se circunscriben a nuestra muestra y no se pueden generalizar por ser una muestra pequeña y formar parte de un estudio exploratorio. Aplicándolos a una población mayor de adolescentes, puede permitir reflejar problemáticas para obtener predictores y variables explicativas de la identidad.

3. En los adolescentes de nuestra muestra, el género no establece diferencias respecto del logro de la identidad. La edad y el grado de instrucción en estos adolescentes, tienen correlación baja pero positiva con respecto al logro de la identidad.

4. Los adolescentes del Instituto presentan un mejor desarrollo en las áreas Personal y Familiar, Educacional y Social que los del Programa.

5. Los adolescentes del programa tienen un mejor logro de identidad, en términos generales. Estas diferencias se pueden explicar en razón de tener más edad y de estar en terapia.

6. La perspectiva Temporal, correlaciona alta y significativamente con la mayoría de los factores componentes de la identidad y está asociada fuertemente a los otros componentes, del área personal en particular. Es importante para la orientación y la prevención de problemas de abuso de drogas en los adolescentes, reforzando su continuidad y proyección vital, ya que el adolescente construye su identidad, como una inteligencia del mundo y de sí mismo, sentido de la vida e identidad, son, mutuamente potenciadores.

\section{REFERENCIAS}

Erikson Erik, (1950) Infancia y Sociedad. Bs. Aires: Paidos.

Erikson Erik (1968) Identidad, Juventud y Crisis Bs. Aires: Paidos.

Muñoz-Rivas, María J., Graña Gómez, José L., Cruzado Rodríguez, Juan (2000) Factores de Riesgo en Drogodependencias: Consumo de Drogas en Adolescentes. Madrid: Sociedad Española de Psicología Clínica, Legal y Forense.

Molla Miguel, (2009). Predictores del Logro de Identidad y Prevención del Abuso de Drogas en Adolescentes. Informe de Investigación presentado en la Plenaria de Investigación de la XXIV Conferencia Mundial de Comunidades Terapéuticas, Lima WFTC

Molla Miguel, (1989). La Identidad en jóvenes de clase media y sus factores condicionantes. (Lima), Informe de Investigación - Consejo Nacional de Ciencia y Tecnología.

Molla Miguel, (1987) La identidad del adolescente, el Test de Identidad (Lima), Revista de Psicología Universidad Católica del Perú. 4 (1) IV

Molla Miguel, y Raguz María (1997) Trastornos de identidad en mujeres con problema de drogas Programa de las Naciones unidas para la fiscalización internacional del Uso de Drogas, Lima: Lluvia

Gossop, Michael; Butrón, Marcela; Molla, Miguel (1993) Altas dosis de cocaína usadas en Perú y Bolivia, The Bethlem Royal Hospital, Londres, Inglaterra, publicado por el Programa de las Naciones Unidas de Lucha contra la Droga.

Ochse, R. \& Plug, C. (1986). Cross-Cultural Investigation of the Validity of Erikson's Theory of personality development. University of South Africa, Pretoria. Journal of Personality and Social Psychology, 50 (6) 1240-1252.

Fecha de recepción: 18 de abril, 2011

Fecha de aceptación: 5 de junio, 2011 